\title{
Endovascular Treatment with an Ultra-thin 4-French Guiding Catheter via the Transfemoral and Transbrachial Routes
}

\author{
- Technical Note-
}

\author{
Hajime TOUHO, Hideyuki OHNISHI, and Jun KARASAWA \\ Department of Neurosurgery, Osaka Neurological Institute, Toyonaka, Osaka
}

\begin{abstract}
An ultra-thin-walled 4-French catheter was used for angiography and as a guiding catheter for the Tracker-18 microcatheter in patients with intracranial dural arteriovenous fistulas (AVFs), spinal dural AVFs, spinal epidural AVF, meningioma, and acute embolic occlusion of major cerebral vessels. The 4-French guiding catheter was introduced through the transfemoral or transbrachial route. The guiding catheter and the guidewire were advanced to the aortic arch and then turned over just above the aortic valves, and finally the catheter was introduced into the external carotid artery or vertebral artery when the transbrachial approach was selected. Images of the intracranial vessels and spinal dural branches obtained were excellent in all cases. The Tracker-18 could smoothly be advanced to the target artery through the 4-French catheter in all patients. Endovascular treatment with the Tracker-18 can be performed using an ultra-thin 4-French guiding catheter, and safely via the transbrachial route.
\end{abstract}

Key words: endovascular treatment, guiding catheter, microcatheter, transfemoral approach, transbrachial approach

\section{Introduction}

Techniques of endovascular treatment including embolization, fibrinolysis, percutaneous transluminal angioplasty, and chemotherapy have recently undergone technical advances. Various microcatheters have been introduced for use in endovascular therapy, including the Tracker-18, which can selectively be advanced to a target artery or vein through a 5-French or larger guiding catheter with thin walls and an inner diameter of $0.97 \mathrm{~mm}$ or larger. ${ }^{3)}$

Guiding catheters used in endovascular treatment are thin-walled and nontapered, and are available in sizes ranging from 5 to 10 French. Guiding catheters of this kind are suitable for introduction of a 2French microcatheter, and should be used with an introducer sheath of 5-French of larger size. ${ }^{3)}$ Using a 5-French or larger guiding catheter for the

Received December 20, 1994; Accepted March 27, 1995 transfemoral approach is thought to be difficult and dangerous in patients with arteriosclerosis obliterance, and sometimes dangerous via the transbrachial route.

In this study, a 4-French thin-walled and nontapered catheter was used for routine performance of cerebral angiography and as a guiding catheter for the Tracker-18 via the transfemoral and transbrachial routes.

\section{Clinical Materials and Methods}

Between May 1 and December 4, 1994, five patients with spinal dural arteriovenous fistulas (AVFs), intracranial dural AVFs, acute embolic occlusion of the right middle cerebral artery, acute embolic occlusion of the basilar artery, or parasagittal meningioma underwent angiography with a 4-French catheter via the transfemoral route, followed by selective embolization or fibrinolysis using the Tracker- 18 (Table 1, Cases 1-5). Three patients with intracranial dural AVFs located at the sigmoid sinus, spinal 
Table 1 Summary of the eight patients treated by endovascular surgery with a 4-French guiding catheter

\begin{tabular}{|c|c|c|c|c|c|}
\hline $\begin{array}{l}\text { Case } \\
\text { No. }\end{array}$ & $\begin{array}{l}\text { Age, } \\
\text { Sex }\end{array}$ & Diagnosis & Approach & $\begin{array}{c}\text { Microcatheter } \\
\text { (Embolic materials) }\end{array}$ & Results \\
\hline 1 & $69, \mathrm{M}$ & $\begin{array}{l}\text { spinal dural AVFs (rt L-5- } \\
\text { S-1) }\end{array}$ & transfemoral & $\begin{array}{l}\text { Tracker-18 embolization } \\
\text { (NBCA) }\end{array}$ & $\begin{array}{l}\text { complete } \\
\text { occlusion }\end{array}$ \\
\hline 2 & $47, F$ & $\begin{array}{l}\text { intracranial dural AVFs } \\
\text { (lt sigmoid sinus) }\end{array}$ & transfemoral & $\begin{array}{l}\text { Tracker-18 embolization } \\
\text { (NBCA) }\end{array}$ & $\begin{array}{l}\text { complete } \\
\text { occlusion }\end{array}$ \\
\hline 3 & $86, \mathrm{M}$ & embolic occlusion of $\mathrm{rt} \mathbf{M}_{1}$ & transfemoral & $\begin{array}{l}\text { Tracker-18 fibrinolysis } \\
(180,000 \text { IU urokinase })\end{array}$ & $\begin{array}{l}\text { complete } \\
\text { recanalization }\end{array}$ \\
\hline 4 & $51, \mathrm{M}$ & It parasagittal meningioma & transfemoral & $\begin{array}{l}\text { Tracker-18 embolization } \\
\text { (Ivalon) }\end{array}$ & $\begin{array}{l}\text { complete } \\
\text { occlusion }\end{array}$ \\
\hline 5 & $90, \mathrm{~F}$ & embolic occlusion of BA & transfemoral & $\begin{array}{l}\text { Tracker-18 fibrinolysis } \\
(360,000 \text { IU urokinase) }\end{array}$ & $\begin{array}{l}\text { incomplete } \\
\text { recanalization }\end{array}$ \\
\hline 6 & $83, \mathrm{M}$ & rt convexity meningioma & transbrachial & Tracker-18 (Ivalon) & $\begin{array}{l}\text { complete } \\
\text { occlusion }\end{array}$ \\
\hline 7 & $74, \mathrm{~F}$ & It $\mathrm{C}-1-\mathrm{C}-2$ epidural AVF & transbrachial & Tracker-18 (NBCA) & $\begin{array}{l}\text { complete } \\
\text { occlusion }\end{array}$ \\
\hline 8 & $77, \mathrm{~F}$ & $\begin{array}{l}\text { intracranial dural AVFs } \\
\text { (rt sigmoid sinus) }\end{array}$ & transbrachial & Tracker-18 (NBCA) & $\begin{array}{l}\text { incomplete } \\
\text { occlusion }\end{array}$ \\
\hline
\end{tabular}

BA: basilar artery, $\mathbf{M}_{1}$ : the first segment of the middle cerebral artery, NBCA: $\mathbf{n}$-butyl cyanoacrylate.

epidural AVF at the high cervical region, or convexity meningioma underwent selective angiography via the transfemoral route resulting in technical failure. However, complete and selective cerebral angiograms could be obtained using the transbrachial approach with turn-over technique (Table 1, Cases $6-8) .^{7,8)}$

The 4-French guiding catheter: The 4-French guiding catheter is constructed of an inner layer of polyurethane-polyamide elastomer containing contrast medium, braid prepared from stainless steel wire mesh, and an outer layer of polyurethane elastomer containing contrast medium. The final 15 $\mathrm{cm}$ of the distal end of the catheter is coated with hydrophilic polymer. The 4-French catheter used in this study was $100 \mathrm{~cm}$ in length with an inner diameter of $1.03 \mathrm{~mm}$, and had a tip $10 \mathrm{~mm}$ in length with a simple primary curve at an angle of approximately 70 degrees to the tertiary curve (Glidecath ${ }^{\mathrm{TM}}$ II; Termo Co., Tokyo). This catheter allowed 360 $\mathrm{mgI}$ of contrast medium to be injected at $10 \mathrm{~mL} / \mathrm{sec}$ (600 psi). The maximum permissible injection pressure was $750 \mathrm{psi}$. The guidewire used was a $0.97-\mathrm{mm}$ Radifocus ${ }^{\circledR}$ guidewire $M$ which was 150 $\mathrm{cm}$ in length (Termo Co.).

Transfemoral approach: A 4-French sheath was introduced into the femoral artery. The 4-French catheter was advanced to the internal carotid artery, external carotid artery, vertebral artery, or an internal iliac artery using conventional techniques. After introduction of the 4-French catheter into the parent artery, the Tracker-18 was selectively advanced to the target artery. Physiological saline containing 10
$\mathrm{IU} / \mathrm{ml}$ heparin was infused through the guiding catheter to prevent embolism.

Transbrachial approach: The patient was placed supine with the right upper extremity extended laterally. The brachial artery was punctured at the elbow and a 4-French sheath was introduced into the right brachial artery. The 4-French guiding catheter was introduced into the cephalic vessels using a turnover technique. ${ }^{8,9)}$ The catheter was advanced over the wire into the aortic arch. The guidewire and the catheter were then advanced and turned over just above the aortic valves, and finally the guidewire was introduced into the right external carotid artery (Cases 6 and 8 ) or the left vertebral artery (Case 7). The catheter was then flushed into those vessels. After introduction of the 4-French guiding catheter into the artery, the Tracker-18 was selectively advanced to the target artery.

In Cases 1, 2, 4, 6, 7, and 8 , embolization with nbutyl cyanoacrylate or calibrated polyvinyl alcohol particles (Ivalon) was successfully achieved. Fibrinolysis with $180,000-360,000$ IU of urokinase was successfully achieved in Cases 3 and 5 (Table 1 ).

\section{Results}

Selective catheterization using the 4-French catheter and four- to six-vessel angiography was completed in all cases except Case 1. Angiograms of the right internal iliac artery were obtained in Case 1. The images of the intracranial vessels and spinal dural branches were excellent in all cases. The Tracker- 18 could smoothly be advanced to the target artery through 
the 4-French guiding catheter in all patients.

No premature ventricular beats were caused by accidental insertion of the catheter and/or the guidewire into the left cardiac ventricle ${ }^{8,99}$ in Cases 68.

\section{Illustrative Cases}

Case 1: A 69-year-old male with slowly progressive paraparesis first noted in April 1984 was admitted to our institute on July 12,1994 . He had severe paraparesis, anesthesia below L-2, and urinary incontinence. Spinal angiography on July 14 revealed spinal dural AVFs at the L-5 to S- 1 level, which were fed by dural branches originating from the right internal iliac artery (Fig. 1 left). After completion of
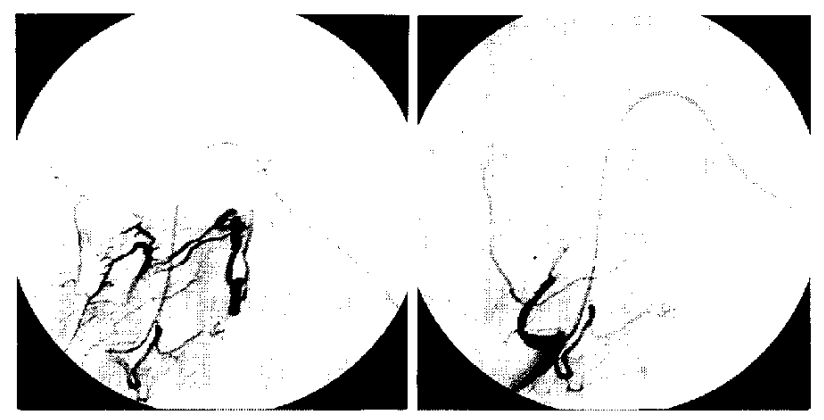

Fig. 1 Case 1. Angiograms (anteroposterior views) showing spinal dural AVFs fed by two dural branches originating from the right internal iliac artery (left), and the completely occluded fistulas following selective embolization (right).
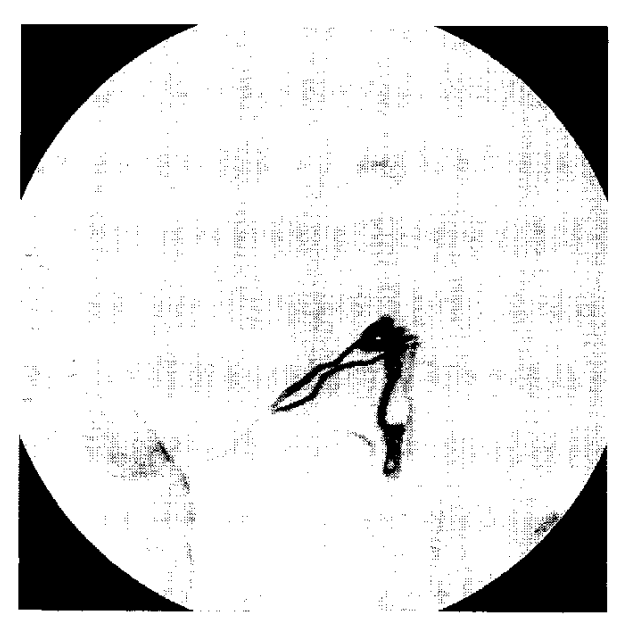

Fig. 2 Case 1. Angiogram (anteroposterior view) showing the Tracker-18 selectively introduced into the radicular artery. spinal angiography, the Tracker-18 was introduced into the radicular artery, which had two dural branches, through the 4-French guiding catheter (Fig. 2). A mixture of n-butyl cyanoacrylate $(0.12$ $\mathrm{ml}$ ) and Lipiodol Ultra-Fluid ${ }^{\circledR}$ (Laboratoire Guerbet, Cedex, France) $(0.12 \mathrm{ml})$, which retards polymerization and is detectable by fluoroscopy, was delivered through the Tracker-18, resulting in complete occlusion of the fistulas (Fig. 1 right). Improvement of sensory disturbance was noted immediately after embolization, and he was discharged on July 19, 1994.

Case 5: A 90-year-old female was admitted to our institute after sudden onset of disturbance of consciousness and quadriparesis. On admission, she had acute respiratory insufficiency, transient atrial fibrillation, quadriparesis, down-beating nystagmus, and somnolence. Cerebral angiography using the 4French catheter disclosed an embolic occlusion of the basilar artery (Fig. 3 left). The Tracker-18 was advanced to the site of occlusion through the 4-French catheter (Fig. 4). After 360,000 IU of urokinase had been infused, recanalization of the basilar artery was obtained (Fig. 3 right). Her neurological state was stable in the intensive care unit after fibrinolysis. However, 4 days after the onset of embolic stroke,
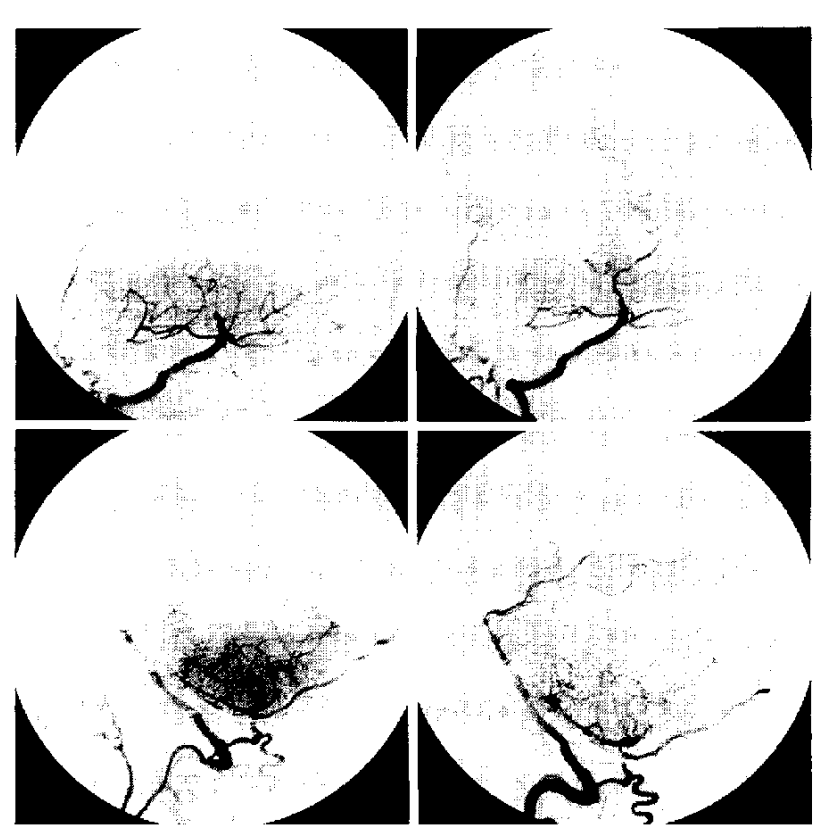

Fig. 3 Case 5. Right vertebral angiograms (upper row: anteroposterior views, lower row: lateral views) disclosing occlusion of the basilar artery (left column), and recanalization after selective intraarterial fibrinolysis with $360,000 \mathrm{IU}$ of urokinase (right column). 


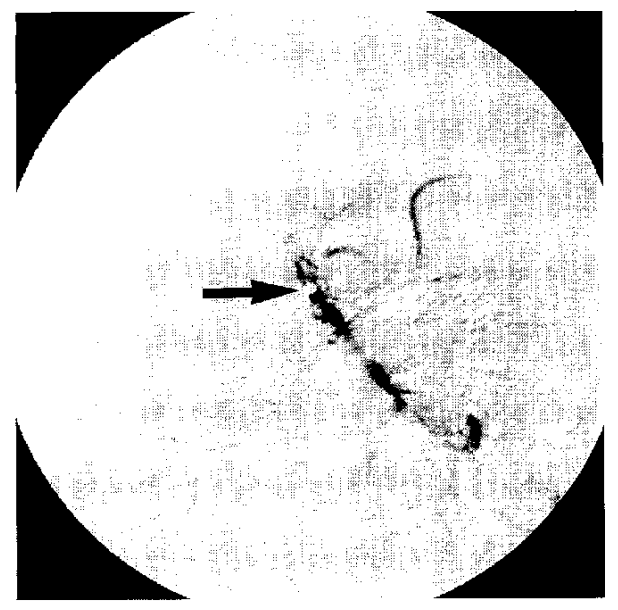

Fig. 4 Case 5. Angiogram (lateral view) showing the Tracker-18 selectively introduced into the basilar artery. The arrow indicates the tip of the Tracker-18.

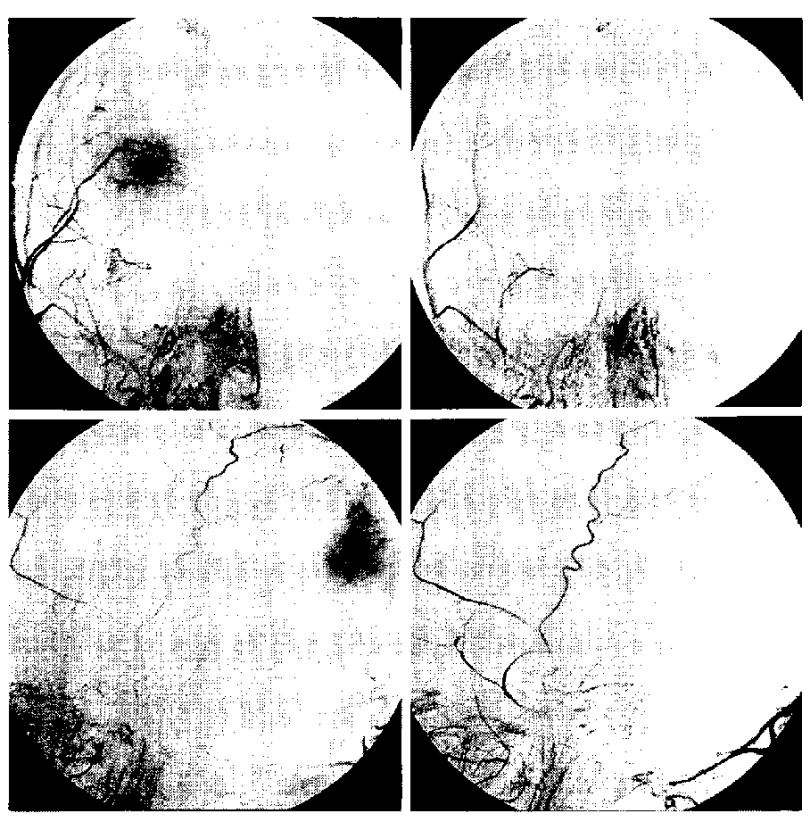

Fig. 5 Case 6. Right external carotid angiograms (upper row: anteroposterior views, lower row: lateral views) demonstrating a right-sided convexity meningioma fed by branches of the right middle meningeal artery (left column), and complete disappearance of the tumor stain just after embolization with polyvinyl alcohol particles (diameter 150-180 $\mu \mathrm{m}$ ) (right column).

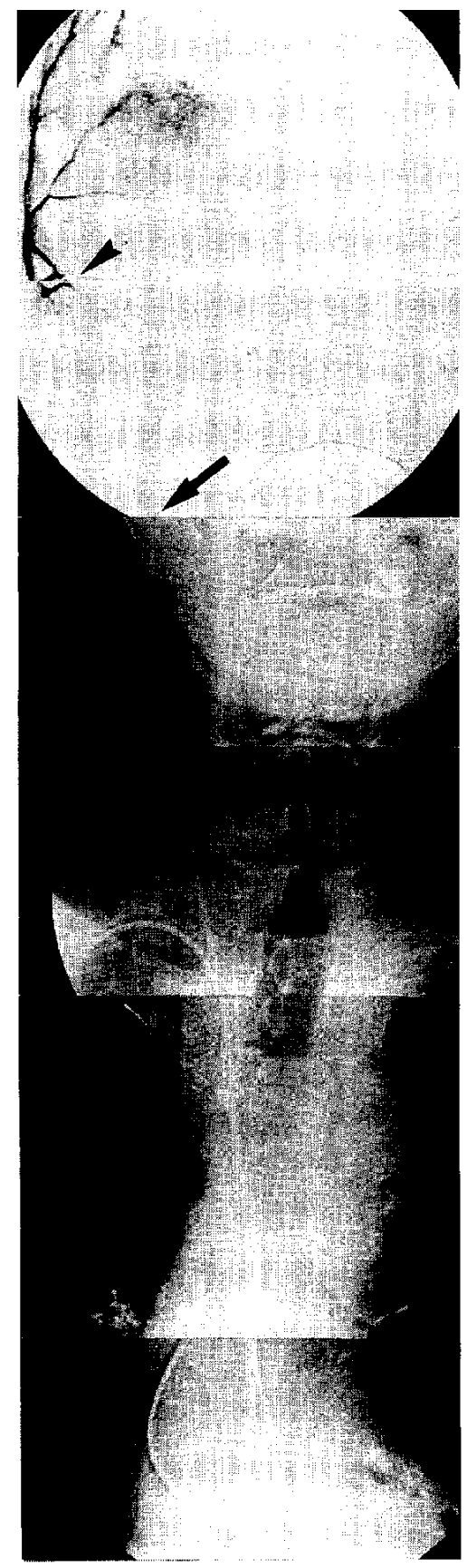

Fig. 6 Case 6. Composite angiogram (anteroposterior view) showing a 4-French guiding catheter advanced to the right external carotid artery via the right transbrachial approach and a Tracker-18 introduced into the posterior branch of the right middle meningeal artery through the guiding catheter. The tip of the guiding catheter (arrow) and the tip of the Tracker-18 (arrowhead) are shown. 
she died of acute myocardial infarction.

Case 6: An 83-year-old male was admitted to our institute with repeated episodes of left-sided clonic convulsions. On admission, he had no focal neurological deficits, but his general condition was not good, and he was malnourished. Computed tomography on admission demonstrated a convexity meningioma in the right parietal lobe. Cerebral angiography could not be achieved via the transfemoral route because of severe atherosclerosis, but was successful via the right transbrachial approach with the 4-French thinwalled catheter and turn-over technique. The meningioma was fed by the right middle meningeal artery (Fig. 5 left). A Tracker-18 was selectively introduced into the posterior branch of the right middle meningeal artery through the 4-French catheter (Fig. 6). The tumor was embolized using Ivalon with particle diameter $150-180 \mu \mathrm{m}$, resulting in complete obliteration of the tumor bed (Fig. 5 right). The postoperative course was uneventful, and he was discharged 13 days after embolization.

\section{Discussion}

This study used a thin-walled 4-French guiding catheter with a 4-French introducer sheath. The angiograms obtained were excellent in quality, and the Tracker-18 could be advanced smoothly to the target arteries. Hemostasis appeared to be more easily achieved with the 4-French sheath than with a 5-French or larger size.

Endovascular treatment via the brachiocephalic vessels is usually performed via the transfemoral route. However, selective catheterization may be difficult in patients with severe aortic atherosclerosis or tortuous brachiocephalic vessels. The transaxillary or transbrachial approach permits catheterization of the cerebral vessels in such patients. ${ }^{1,2,4-6)}$ Selective angiography of all four vessels via one route is possible using a modified Simmons catheter. ${ }^{9}$ However, obtaining selective angiograms of the internal carotid and external carotid arteries is impossible because of the short tip of the modified Simmons catheter. The turn-over technique for selective cerebral angiography via the transfemoral and transbrachial routes was recently described. ${ }^{7,8)}$ A 4French, 120-cm long catheter (BTOHO-120S; Cathex Co., Inc., Tokyo) was previously used with the turn-over technique via the transbrachial route. However, neither the Tracker-18 nor the Tracker-10 could be passed through the catheter.

In this study, a 4-French ultra-thin catheter was introduced as a guiding catheter for the Tracker- 18 . The Tracker-18 passed smoothly through the ultra- thin catheter. The Tracker-18 could be advanced easily to a target vessel through the 4-French guiding catheter which was turned over just above the aortic valves, because advancing and pressing the Tracker18 into intracranial vessels against the valves presented no difficulty. Access to extra- and intracranial lesions with the Tracker catheter was easy and embolization through the microcatheter could be successfully performed. Cerebral angiography via the 4-French guiding catheter during embolization was also possible. However, the angiograms did not visualize the target lesion clearly because the distance between the inner lumen of the guiding catheter and the outer surface of the Tracker-18 was small. This is a disadvantage of using this catheter.

The 4-French guiding catheter can be used for endovascular treatment with the Tracker-18 via the transfemoral route. Endovascular treatment of various extra- and intracranial lesions in patients, especially the elderly, can be easily and safely performed using the 4-French ultra-thin guiding catheter and turn-over technique via the transbrachial approach.

\section{References}

1) Adams PS Jr, Roub LW: Outpatient angiography and interventional radiology: Safety and cost benefits. Radiology 151: 81-82, 1984

2) Becker GJ, Hicks ME, Holden RW, Edwards MK, Jackson VP, Bendick PJ: Screening for occlusive vascular disease with intraarterial DSA: Preliminary experience with a high-flow 4-F catheter. Radiology 153: 823, 1984

3) Dion JE: Principles and methodology, in Viñuela F, Halbach VV, Dion JE (eds): Interventional Neuroradiology. Endovascular Therapy of the Central Nervous System. New York, Raven Press, 1992, pp 115

4) Hanafee W: Axillary artery approach to carotid, vertebral, abdominal aorta, and coronary angiography. Radiology 81: 559-567, 1963

5) Newton TH: The axillary artery approach to arteriography of the aorta and its branches. Amer $J$ Roentgen 89: 275-283, 1963

6) Roy P: Percutaneous catheterization via the axillary artery. A new approach to some technical roadblocks in selective arteriography. Amer J Roentgen 91: 1-18, 1965

7) Touho H, Karasawa J, Ohnishi H, Nakase H, Watabe Y, Yamada K, Sato N, Seno M, Takaoka M, Komatsu T: Turn over technique for selective cerebral angiography. Neuroradiology 36: 123-124, 1994

8) Touho H, Karasawa J, Ohnishi H, Nakase H, Watabe Y, Yamada K, Seno M, Sato N, Takaoka M, Komatsu T: Transbrachial approach with turn over technique for selective cerebral angiography: Technical note. 
Neurol Med Chir (Tokyo) 33: 716-718, 1993

9) Touho H, Karasawa J, Shishido H, Tazawa $T$, Yamada K, Kobayashi K, Asai M, Yasue H, Kagawa M: Transbrachial artery approach for selective cerebral angiography in outpatients. AJNR 9: 334336,1988
Address reprint requests to: $\mathrm{H}$. Touho, M.D., Department of Neurosurgery, Osaka Neurological Institute, 2-623 Shonai-takara-machi, Toyonaka, Osaka 561, Japan. 